\title{
Design of UAV wireless power transmission system based on coupling coil structure optimization
}

\author{
Yixin Yan, Wan Shi and Xiaobing Zhang*
}

\begin{abstract}
With the development of unmanned aerial vehicle (UAV), it has been used in many fields, such as aerial photography, military, and transportation. However, it is a challenge for charging due to its short battery life. Wireless charging has been considered as a good UAV solution. In this paper, we proposed a wireless power transmission system (WPT) through inductive coupling and analyzed the power efficiency of the inductive coupling. The WPT included a charging station, which can provide energy through an induction coil array, and a portable energy receiving device mounted on the base of the UAV. Moreover, the power efficiency was analyzed though the equivalent circuit of WPT and the mathematical model of the power efficiency was established. The results showed that the WPT can transfer energy to UAV with high power efficiency within a $30 \mathrm{~mm}$ lateral displacement range.
\end{abstract}

Keywords: Unmanned aerial vehicle, Wireless charging, Magnetic coupling resonance

\section{Introduction}

In recent years, the performance of unmanned aerial vehicle (UAV) is gradually optimized. Its application is expanded from military to civil use [1, 2], scientific research, and other fields [3-5]. UAV can be equipped with many payloads, such as cameras, sensors, positioning systems [6-8]. These loads are often powered by high energy density lithium batteries, which can only last for 20-40 min [9-11]. The lithium battery limits the length of UAV possible running length [12-15]. It is necessary for UAV continuously conduct inspections and detection under working condition [16-18]. Therefore, it is important to elongate the running time of UAV [19]. Three potential methods are proposed to increase the flight time of UAV. The first method is to build up a new type battery with higher UAV capacity [20], but this method will cause unexpected high body weight of

\footnotetext{
* Correspondence: zhang_xiaobing123@126.com Electronic Measurement and Control Laboratory, School of Measurement Control and Communication Engineering, Harbin University of Science and Technology, Harbin 150080, People's Republic of China
}

UAV. It will indirectly reduce the loading efficiency [2123]. The second method is to set up a base station, and the UAV can exchange battery automatically in the base station. This method can solve the problem of endurance of UAV [24]. However, this proposal is not practical because of its high cost and the complexity of the algorithm. The third known method is to build a charging pile on UAV that can recharge the UAV directly.

There are two ways to charge UAV: (1) build direct contact between the charging pile and UAV to charge UAV directly. Although the transmission efficiency of this direct contact pile model is high [25], the unpredictable serious environmental factors will destabilize the entire system in the air. (2) Use wireless power transmission (WPT) technology with the coupling resonance between the coils to recharge UAV [26-28]. There are several not ideal factors that need to be taken into consideration. (A) The size of coupling device. (B) The actual load of UAV. (C) The energy safety. The most important point is that WPT must consider the efficiency of power transmission. The inaccurate landing of

\section{Springer Open}

() The Author(s). 2020 Open Access This article is licensed under a Creative Commons Attribution 4.0 International License, which permits use, sharing, adaptation, distribution and reproduction in any medium or format, as long as you give appropriate credit to the original author(s) and the source, provide a link to the Creative Commons licence, and indicate if changes were made. The images or other third party material in this article are included in the article's Creative Commons licence, unless indicated otherwise in a credit line to the material. If material is not included in the article's Creative Commons licence and your intended use is not permitted by statutory regulation or exceeds the permitted use, you will need to obtain permission directly from the copyright holder. To view a copy of this licence, visit http://creativecommons.org/licenses/by/4.0/. 
this system will lead to misaligned of coils, which will reduce the efficiency of WPT. There are many methods are introduced to solve this problem. (A) Use automatic alignment landing procedure, image processing technology, and position technology to enhance the landing accuracy of UAV [29-31]. (B) Move transmitting coils to align with airborne receiving coils through both the internal mechanical structure of the charging pile and the external sensor to improve the transmission efficiency $[32,33]$. The position and sensor detection of UAV is often affected by various environmental factors, which lowers the landing accuracy of UAV [34].

Those methods mentioned above cannot effectively solve the problem of low efficiency caused by the misalignment of the primary coil and the secondary coil. Solving the problem of low charging efficiency due to misaligned coupling coils is the main challenge for enabling patrol drones to constantly check the workspace. The major point is to find a way to increase the transmission power efficiency when the coupling coil is misaligned. In this research, a new type of coupling coil is designed, which can improve the power and efficiency of WPT even if the coupling device is offset.

The main contributions of this paper include:

1. The compensation circuits are analyzed in principle, and then formulas for those compensation circuits are derived. According to those derived formulas, a Series-Series compensation circuit is selected. And then, the inductance and capacitance value of this compensation circuit is calculated.

2. This research builds a new type of coupling coil array, and demonstrates its advantages by analyzing data collected through experiments.

The rest of this paper is organized as follows. Section 2 discusses the overall scheme design of WPT system. WPT system of UAV is designed and simulation results of the system are given in Section 3. The hardware of the system is designed in Section 4. Section 5 concludes the paper with summary.

\section{The proposed scheme}

\subsection{System model}

The WPT system of UAV is mainly composed of transmitter and receiver. The primary coil is arranged on the charging pile, and the secondary coil is arranged on the UAV, and lithium battery is commonly used as energy source to provide voltage UAV. The wireless charging system is built to use coil to transmit energy to the battery. For the general UAV, its power system is generally composed of three to six lithium batteries with capacity between 3 and 10Ah, which can provide up to $11.1 \mathrm{~V}$ to $22.2 \mathrm{~V}$. This type of lithium battery can be quickly fully charged in an hour. Coil coupling is an important parameter, which can be improved by reducing air gap, to improve the charging efficiency. The secondary coil that set on the landing sled of UAV can reduce the vertical air gap between the primary and secondary coil, to improve the transmission efficiency. The secondary coil at the receiver is designed as a small circular coil to facilitate the placement of the UAV above the sled. The primary coil of the transmitter is designed as an independent coil array as shown in Fig. 1, and the primary coil is placed on the charging pile.

The independent coil array design of the primary coil is essential, because it ensures enough coupling at all landing positions. The working principle of the independent coil array is that when the UAV lands, the Bluetooth sensor on the charging pile detects the Bluetooth signal of the UAV, monitors whether the UAV has landed on principle of the independent coil array is that when the UAV lands, and then the controller opens the charging area and the primary coil is coupled to the secondary coil, and finally the UAV is charged through the primary coil.

In a typical WPT charging system, the voltage is adjusted before the charging link of the receiver. The voltage is adjusted at the transmitter region first to reduce the body weight and occupied space of system. The structure diagram of the whole WPT system is shown in Fig. 2. The voltage at the transmitter is adjusted using a direct current (DC)/DC converter before inverter, and then converts $\mathrm{DC}$ to alternating current $(\mathrm{AC})$ through inverter, and then low frequency part of the $\mathrm{AC}$ is filtered by a high-pass filter circuit, and the high frequency $\mathrm{AC}$ is transmitted through the wireless coupling between the coils. The receiver of the UAV receives energy

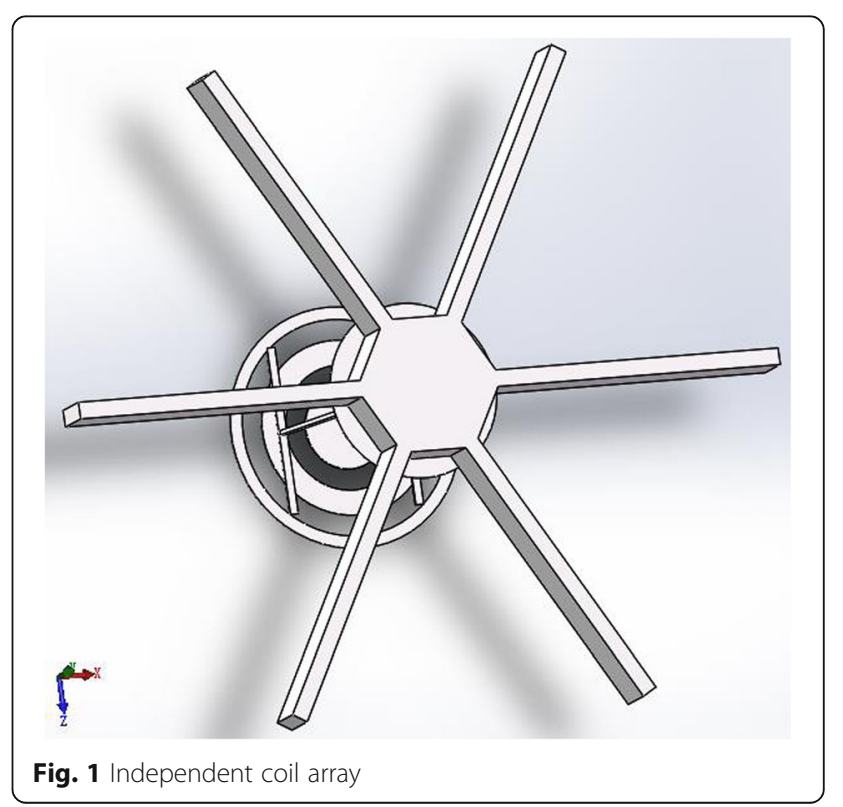




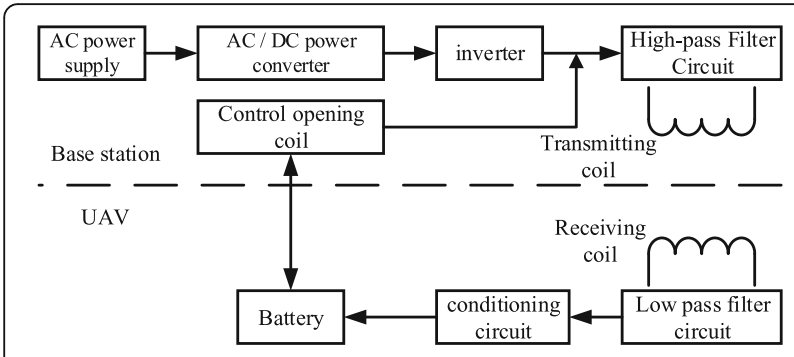

Fig. 2 Structure diagram of WPT system

through resonance magnetic coupling. Then the voltage of battery recovers through rectifying, filtering, and compensating circuits. In this way, the UAV can work efficiently and transmit medium range wireless power.

\subsection{WPT equivalent circuit}

WPT technology is a kind of near-field energy transfer method, which transfers energy from power supply to load through magnetic resonance coupling coil without direct ground electrical contact. The loose coupling transformer is composed of two coils to build the magnetic resonance coupling coil of WPT. Based on the equivalent circuit theory, this paper models and analyzes the WPT of magnetic coupling resonance, and optimizes the charging distance, power, and efficiency parameters of UAV. The equivalent circuit of the whole WPT system is shown in Fig. 3. There are coil self-inductance $L_{1}$ and $L_{2}$ between the coupling coils, mutual inductance $M$, and coil self-resistance $R_{1}$ and $R_{2}$. Self-inductance, mutual inductance, and self-resistance cause the loss of the whole system. In order to reduce the AC loss caused by skin effect and proximity effect, the coupling coil shall be wound by copper stranded wire. The coupling coefficient $K$ of the coil is:

$$
K=\frac{M}{\sqrt{L_{1} L_{2}}}
$$

A capacitor compensation network is added to the transmitter and receiver to obtain continuously resonance and improve the power transmission performance of the system. As shown in Fig. 3, this circuit is a typical topology with Series-Series (S-S) compensation. At the transmitter, a full bridge inverter composed of four MOSFETs (Q1, Q2, Q3, Q4) is used to convert the DC voltage into the $\mathrm{AC}$ voltage $\mathrm{V} 1$. At the receiver, the high-frequency voltage V2 transmitted through the coupler is first rectified by a full bridge rectifier composed of D1, D2, D3, and D4. The output voltage after rectification is filtered by a low-pass filter composed of L3 and C3, and finally charges the battery.

To facilitate the calculation and extraction of lumped parameters, the circuit is simplified, as shown in Fig. 4. In the figure, $R_{S}$ is the resistance of the power supply, $R_{L}$ is the load resistance, $R_{1}, R_{2}$ represent the internal resistance of each coil, $L_{1}, L_{2}$ represent the equivalent inductance of the coil, $C_{1}, C_{2}$ represent the compensation capacitance of the circuit, and $M$ represents the mutual inductance between the primary and secondary coils.

In Fig. 4, the self-impedance of the main circuit is expressed as $Z_{11}$, the expression is $Z_{11}=R_{S}+R_{1}+j\left(\omega L_{1}\right.$ $\left.-1 / \omega C_{1}\right)$, the self-impedance of the secondary circuit is expressed as $Z_{22}$, and the expression is $Z_{22}=R_{2}+R_{L}+$ $j\left(\omega L_{2}-1 / \omega C_{2}\right)$. The voltage equation of main circuit and secondary circuit can be written as follows:

$$
\left\{\begin{array}{c}
I_{1}\left(Z_{11}-j \omega M\right)+\left(I_{1}-I_{2}\right) j \omega M=U S \\
I_{2}\left(Z_{22}-j \omega M\right)+\left(I_{2}-I_{1}\right) j \omega M=0
\end{array}\right.
$$

From Eq. (2), the current equations of the main circuit and the secondary circuit can be deduced as follows:

$$
\left\{\begin{aligned}
I_{1} & =\frac{Z_{22} U_{S}}{Z_{11} Z_{22}+(\omega M)^{2}} \\
I_{2} & =\frac{j \omega M U_{S}}{Z_{11} Z_{22}+(\omega M)^{2}}
\end{aligned}\right.
$$

It can be seen from the expression of the secondary circuit current in formula (3) that the induced electromotive force is related to the angular frequency $\omega$ and the mutual inductance coefficient $M$ of the coil. Only when the imaginary part of the current expression of the secondary circuit is zero, the circuit is in resonance state, the current of the secondary circuit reaches the maximum value, and the load obtains the maximum power.

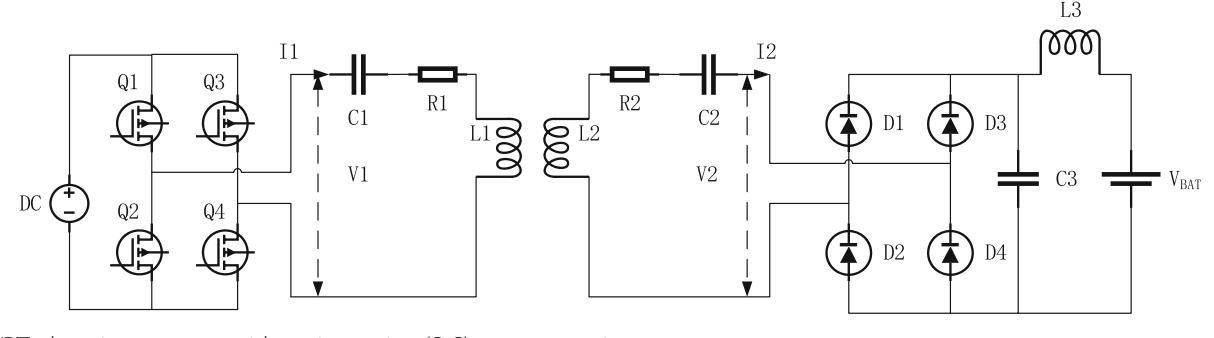

Fig. 3 Circuit of WPT charging system with series-series (S-S) compensation 


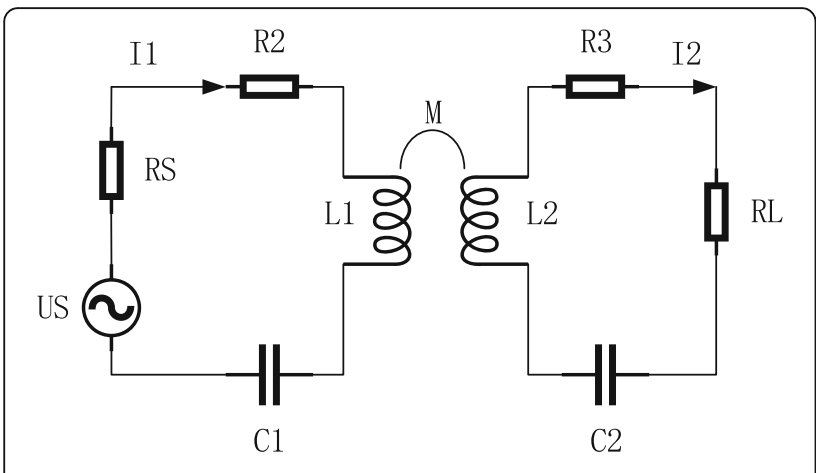

Fig. 4 Simplified circuit with S-S compensation

The receiving power of the load and the transmission efficiency of the system can be expressed by the following formula:

$$
\begin{gathered}
P_{L}=I_{2}^{2} R_{L}=\left(\frac{\omega M U_{S}}{Z_{11} Z_{22}+(\omega M)^{2}}\right)^{2} \\
\eta=\frac{P_{L}}{P_{I N}} \times 100 \%=\frac{\omega M^{2} R_{L}}{Z_{22}\left(Z_{11} Z_{22}+(\omega M)^{2}\right)} \\
=\frac{\omega M^{2} R_{L}}{R_{2}+R_{L}}
\end{gathered}
$$

As shown in Eq. (5), increasing the resonant frequency $\omega$ or the mutual inductance coefficient $M$ can improve the transmission efficiency of the system. If only increasing the resonant frequency will lead to the skin effect of the conductor, resulting in higher energy loss and lower efficiency, but only increasing the mutual inductance of the coupling coil will lead to the reduction of the effective transmission distance. Therefore, it is necessary to introduce two other key parameters: quality factor $Q$ and coupling factor $K$ to discuss the transfer performance.

According to the circuit theory, the quality factors of the coupling coil, $Q_{1}$ and $Q_{2}$, are as follows:

$$
\begin{aligned}
& Q_{1}=\frac{\omega L_{1}}{R_{1}+R_{S}} \\
& Q_{2}=\frac{\omega L_{2}}{R_{2}+R_{L}}
\end{aligned}
$$

The expressions of power and efficiency can be obtained by introducing formulas (1), (6), and (7) into Eqs. (4) and (5):

$$
\begin{aligned}
& P_{L}=\frac{U_{S}^{2} R_{L}}{\left(R_{1}+R_{2}\right)\left(R_{2}+R_{L}\right)} \frac{k^{2} Q_{1} Q_{2}}{\left(1+k^{2} Q_{1} Q_{2}\right)^{2}} \\
& \eta=\frac{R_{L}}{R_{2}+R_{L}} \frac{k^{2} Q_{1} Q_{2}}{\left(1+k^{2} Q_{1} Q_{2}\right)}
\end{aligned}
$$

The MATLAB simulation software is used to simulate the received power and transmission efficiency, and the curves of receiving power and transmission efficiency with respect to quality factor $Q$ and coupling factor $K$ are obtained, as shown in Figs. 5 and 6.

As shown in Figs. 5 and 6, the transmission performance can be effectively improved only by making $K, Q_{1}$, and $Q_{2}$ achieve their maximum value in the resonant magnetic coupling system with constant resonant frequency. However, it is difficult to improve the system

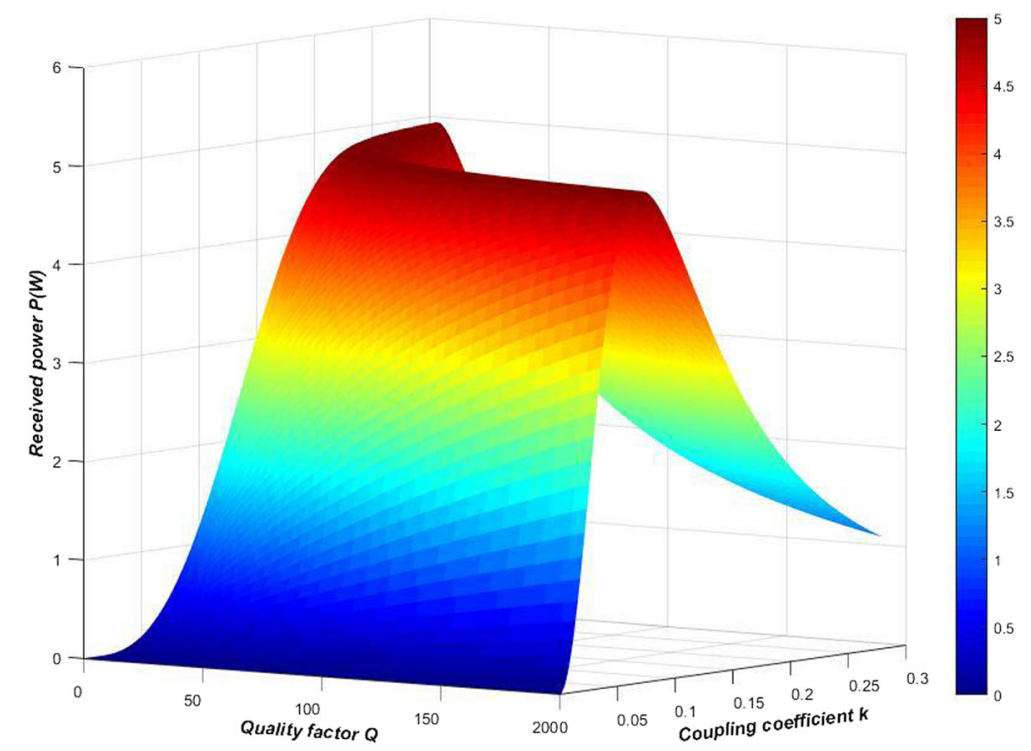

Fig. 5 Influence curve of quality factor and coupling factor on received power 


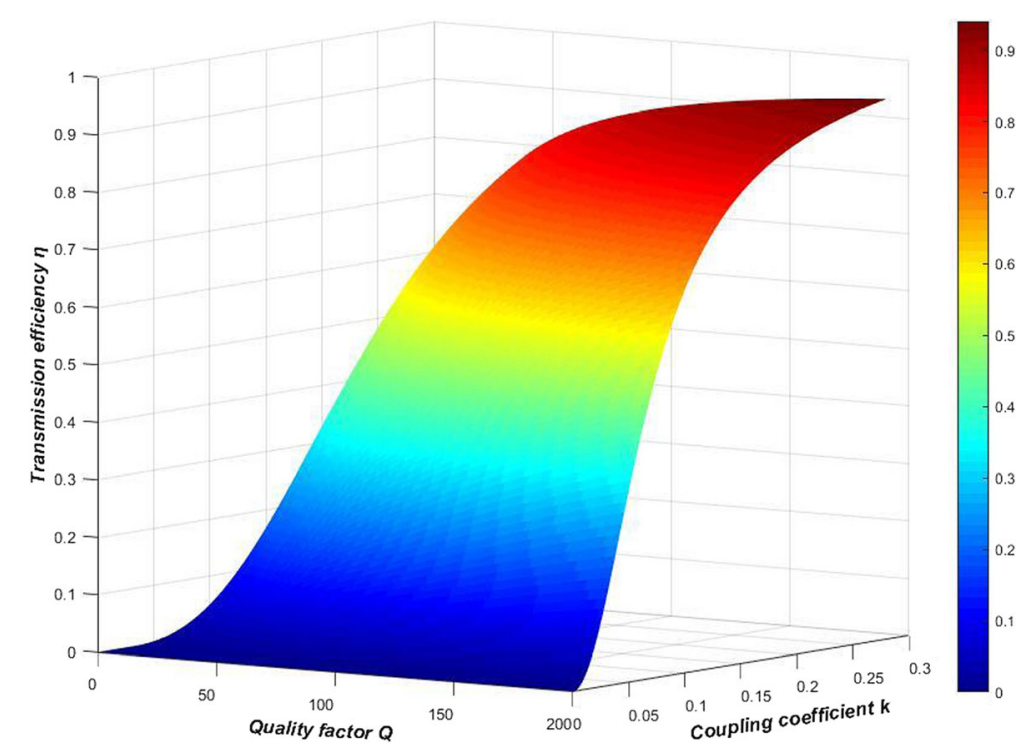

Fig. 6 Influence curve of quality factor and coupling factor on transmission efficiency

performance by increasing $K$, because the transmission medium is basically air. The system performance can be improved in another way. Instead of increasing the coupling coefficient by increasing the quality factor of the coil, the effective transmission distance is greatly improved, while receiving efficiency and transmission efficiency are improved. According to formula (6) and (7), it can be seen that the quality factor is related to the working frequency $\omega$, the coil self-induction $L$, and the coil internal resistance $R$, and it does not change with other parameters such as the relative position between the coupling coils. Therefore, this paper focuses on the design of high-quality factor coil and the solution of coil misalignment during UAV charging.

\subsection{Selection of compensation circuit}

Coupling coil is one of the key devices in wireless transmission. In the coupling coil, the air gap between the transmitting coil and the receiving coil is large. In general, the coupling coefficient between the two coils is not too large, so the efficiency of power transmission is extremely low under the excitation of $50 \mathrm{~Hz}$ signal source. In order to make the radio transmission efficiency and power meet the requirements of use, capacitors are added to the primary and secondary circuits for compensation, and the inductance and capacitance values of the primary and secondary circuits are adjusted at the same time, so that the two circuits have the same resonance frequency, to achieve efficient transmission.
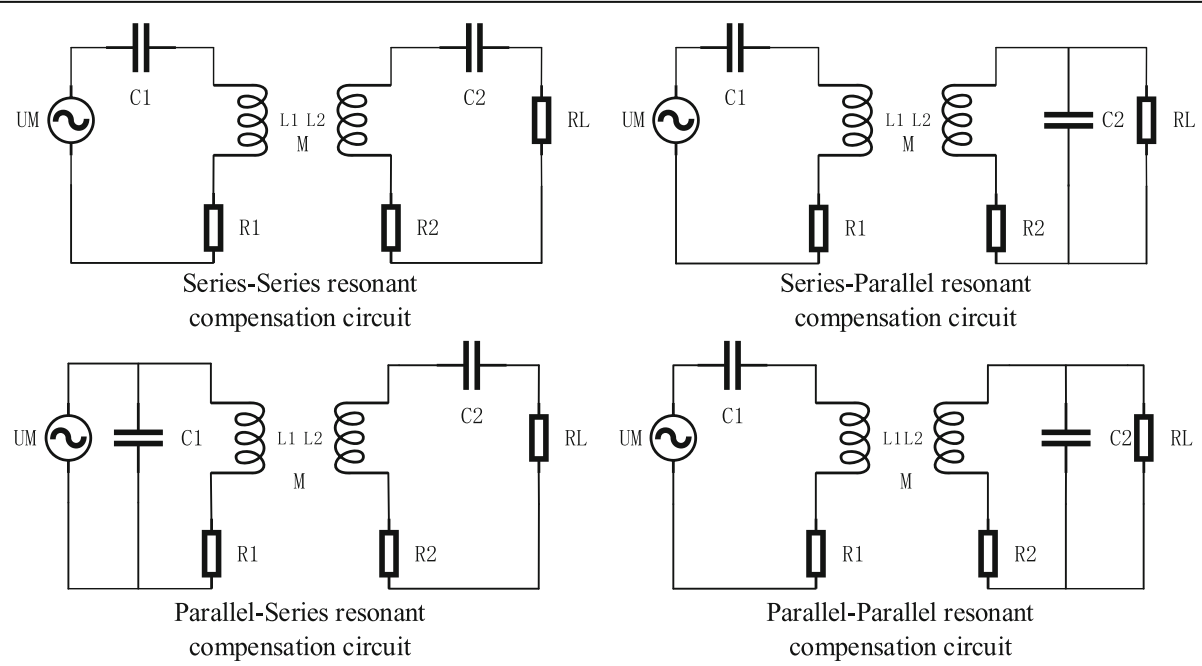

Fig. 7 Four basic resonant compensation circuits 
Table 1 Total impedance of four kinds of resonant compensation circuits

\begin{tabular}{ll}
\hline Compensation circuit & Input impedance \\
\hline S-S & $j\left(\omega L_{1}-\frac{1}{\omega C_{1}}\right)+\frac{\omega^{2} M^{2}}{R_{L}+j\left(\omega L_{2}-\frac{1}{\omega C_{2}}\right)}$ \\
S-P & $j\left(\omega L_{1}-\frac{1}{\omega C_{1}}\right)+\frac{\omega^{2} M^{2}}{j \omega L_{2}+\frac{R_{L}}{j \omega C_{2} R_{L}+1}}$ \\
P-S & $\frac{1}{\frac{1}{j \omega L_{1}+\frac{\omega^{2} M L^{2}}{R_{L}+j\left(\omega L_{2}-\frac{1}{\omega C_{2}}\right)}+j \omega C_{1}}}$ \\
P-P & $\frac{1}{j \frac{\omega^{2} M^{2}\left(1+j \omega C_{2} R_{L}\right)}{j \omega \omega L_{1}}+j C_{1}}$ \\
\hline
\end{tabular}

At present, the resonant compensation circuit is mainly divided into four types according to the connection mode of capacitor: Series-Series (S-S) resonant compensation circuit, Series-Parallel (S-P) resonant compensation circuit, Parallel-Series (P-S) resonant compensation circuit, and Parallel-Parallel (P-P) resonant compensation circuit. The resonance compensation circuits are shown in Fig. 7.

Among the four structures, $U_{M}$ is the voltage source in the charging system; $C_{1}, L_{1}$, and $R_{1}$ are the compensation capacitance, coil self-induction, and internal resistance in the primary circuit. $C_{2}, L_{2}$, and $R_{2}$ are the compensation capacitance, coil self-induction, and internal resistance in the secondary circuit, and $R_{L}$ is the equivalent load of the system.

Firstly, the qualitative analysis of these four basic resonant compensation circuits is carried out. It is assumed that the inductance and capacitance are connected in series; when resonance occurs, the resistance of the wires in the circuit and the equivalent resistance of the resonance are very small. Therefore, the whole compensation circuit can be regarded as a short circuit. The current flowing through the coil will be very large, but the voltage will not have a voltage drop, so the whole circuit can be equivalent to a voltage source and a load in series. It is assumed that the inductance and capacitance are connected in parallel; when resonance occurs, the impedance of the compensation circuit is infinite, the current flowing through the coil will be very small, and the current of the capacitance will cancel the

Table 2 Capacitance values of four compensation circuits

\begin{tabular}{lll}
\hline $\begin{array}{l}\text { Compensation } \\
\text { circuit }\end{array}$ & $\begin{array}{l}\text { Capacitance of transmitting } \\
\text { coil } C_{1}\end{array}$ & $\begin{array}{l}\text { Capacitance of } \\
\text { receiving coil } C_{2}\end{array}$ \\
\hline S-S & $\frac{1}{\omega^{2} L_{1}}$ & $\frac{1}{\omega^{2} L_{2}}$ \\
S-P & $\frac{\omega^{2} L_{2}^{2}}{\omega^{4} L_{1} L_{2}^{2}-\omega^{4} M^{2} L_{2}}$ & $\frac{1}{\omega^{2} L_{2}}$ \\
P-S & $\frac{\omega^{2} L_{2}^{2}}{\frac{\omega^{2} M^{2}}{R_{L}^{2}}+\omega^{2} L_{1}^{2}}$ & $\frac{1}{\omega^{2} L_{2}}$ \\
P-P & $\frac{\omega L_{1}+\frac{M^{2}}{L_{2}}}{\frac{M^{4} R_{L}^{2}}{L_{2}^{4}}+\left(\frac{\omega^{2}}{L_{2}}+\omega L_{1}\right)^{2}}$ & $\frac{1}{\omega^{2} L_{2}}$ \\
& & \\
\hline
\end{tabular}

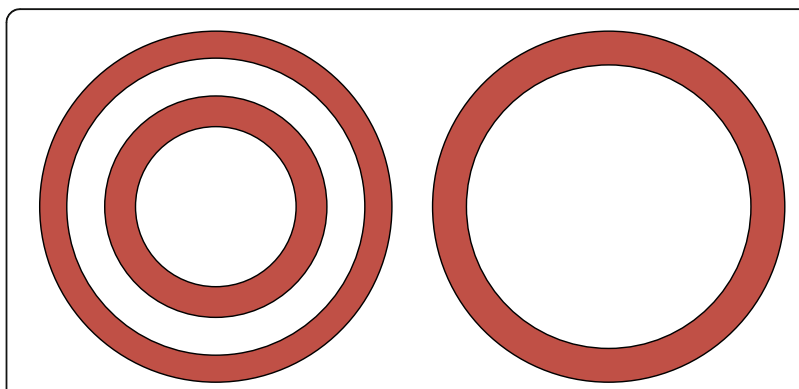

Fig. 8 Structure diagram of asymmetrical coupling coil. a Transmitting coil. b Receiving coil

inductance current, so the whole circuit can be equivalent to a current source and load in parallel.

Then the quantitative analysis of the four basic resonant compensation circuits is carried out. In order to simplify the circuit model, the internal resistances of wires, inductors and capacitors in the circuit are ignored, and the ratio of turns of transmitting coil and receiving coil are assumed to be 1:1.

After derivation of the formula, the total impedance of the four basic resonant compensation circuits is shown in Table 1.

In order to maximize the output power of wireless charging system, the reactive power on inductance and capacitance must be minimized. Therefore, it is necessary to make the overall impedance of the compensation circuit show pure resistance. According to the total impedance in the above table and the assumed $L_{1}$ and $L_{2}$, when the system unit power factor is reached, the capacitance value in the wireless charging system can be calculated, as shown in Table 2.

From the formula of resonance capacitance, it can be seen that when the load $R_{L}$ and $M$ change, in order to make the system reach the unit power factor, the resonant compensation circuit is S-P resonant compensation circuit, P-S resonant compensation circuit, and the P-P resonant compensation circuit, and their capacitance values need to be changed. Only when S-S resonant compensation circuit is used, it will not change with the change of load and coupling coefficient. Therefore, considering the actual wireless charging system, S-S resonant compensation circuit is more suitable for UAV wireless charging.

Table 3 Coil structure parameters

\begin{tabular}{lll}
\hline Section & Transmitting coil & Receiving coil \\
\hline Turns/N & 25 & 25 \\
Internal diameter/mm & 150,260 & 150 \\
External diameter/mm & 210,320 & 210 \\
Inductance value/mH & 1.4192 & 0.3723 \\
Capacitance value/nF & 178.48 & 680.37 \\
\hline
\end{tabular}




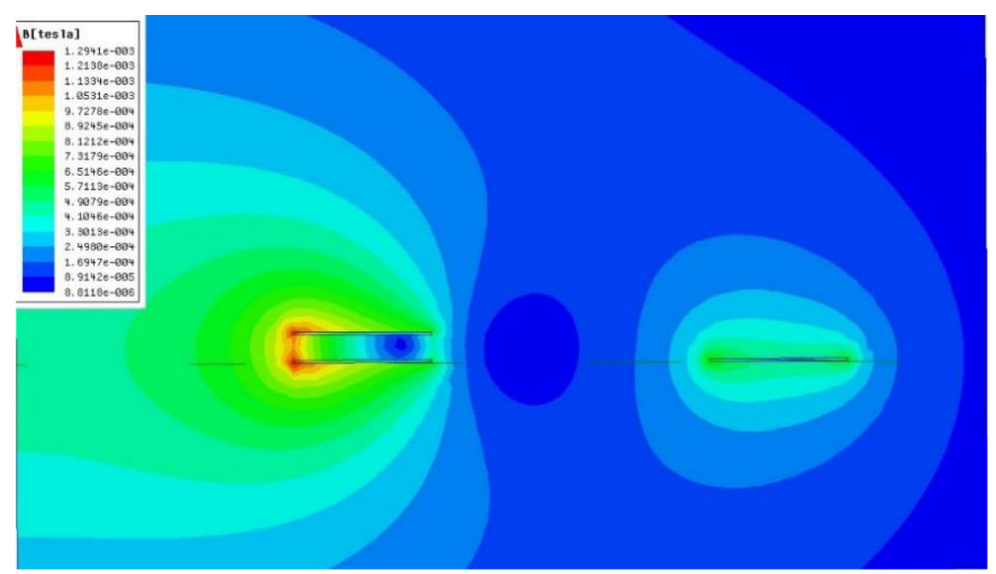

(a)

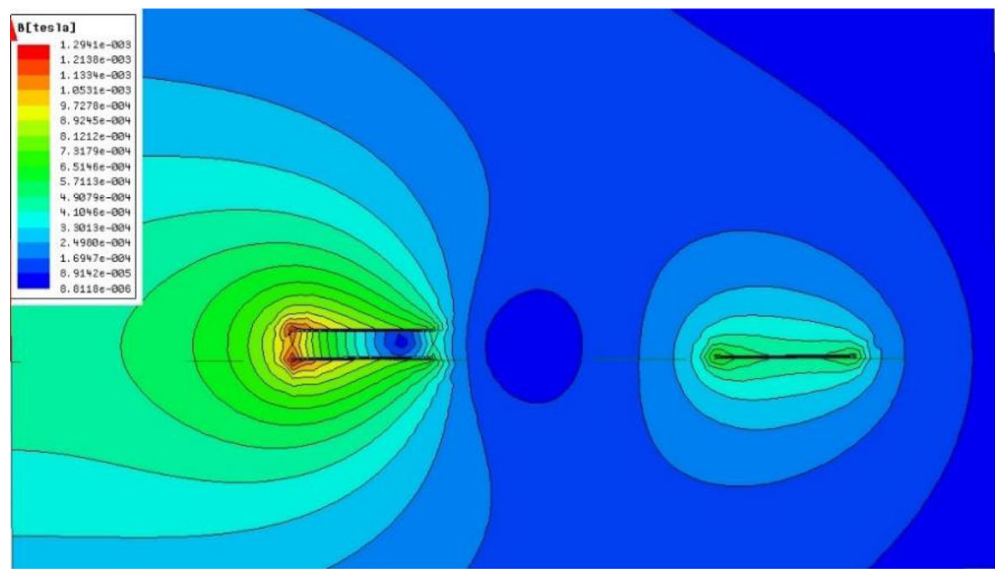

(b)

Fig. 9 Magnetic field distribution of asymmetric coupling coil structure. a Magnetic field without equipotential line. b Magnetic field with equipotential line

\subsection{Design of coupling coil}

The coupling mechanism mainly includes the transmitting coil inside the charging pile and the receiving coil at the bottom of the UAV, which is the core part of the magnetic coupling resonance WPT system. According to the investigation of UAV on the market, the weight of UAV is generally $4-5 \mathrm{~kg}$. Because of the requirement of the total weight of UAV airborne equipment, the size of receiving coil shall be as small as possible and the weight should be as light as possible. Therefore, the plane spiral coil is selected in this paper, which has the advantages of small volume, easy winding, and assembly.

The design of coupling coil shall consider the following capacities:

1. Because of the small size of the receiving coil, it is difficult to realize the precise coupling between the coupling coils when landing. Therefore, the size of transmitting coil shall be as large as possible in

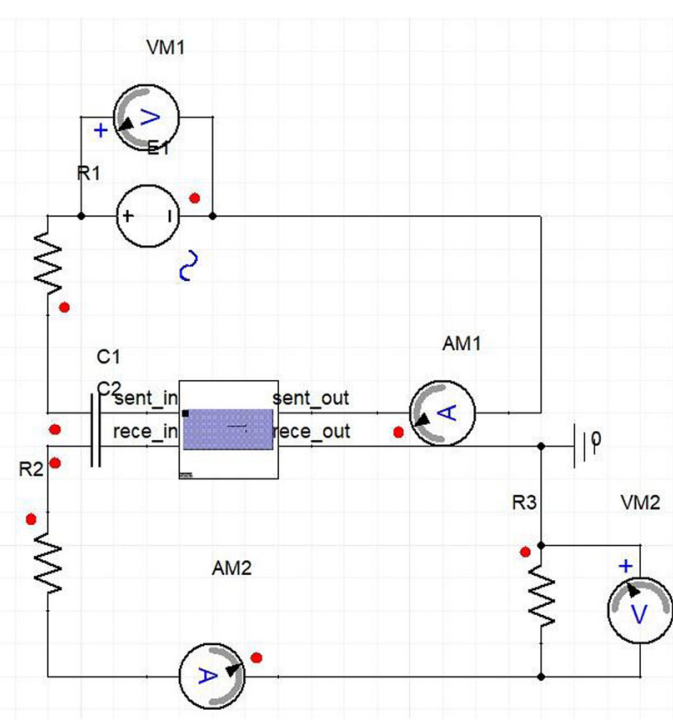

Fig. 10 Circuit simulation diagram on simplorer 
Table 4 System parameters

\begin{tabular}{ll}
\hline Parameter & Value \\
\hline Receiving power & $65.77 \mathrm{~W}$ \\
Transmission efficiency & $62.44 \%$ \\
Working frequency & $162 \mathrm{kHz}$ \\
Coupling coil spacing & $40 \mathrm{~mm}$ \\
Quality factor of transmitting coil & 204.1 \\
Quality factor of receiving coil & 134.4 \\
\hline
\end{tabular}

order to enlarge the rechargeable area relative to the receiving coil.

2. Considering the accuracy of the GPS positioning system of UAV, the coupling coil is difficult to achieve accurate docking. Therefore, the coil array can be used in the transmitter to greatly increase the intensity and uniformity of the emission magnetic field, to achieve better transmission performance.

3. In order to improve the effective transmission distance of WPT, the higher resonance frequency coil shall be selected. The coil shall be tightly wound with multiple strands of Leeds to reduce the skin effect.

4. Considering the number of coil turns, it shall not be too much. If the number of coil turns is too much, the mutual inductance will be saturated, and the volume of the coil will be too large.

In consideration of the above requirements, the transmitting coil is designed as a plane concentrated coil array composed of two concentric coils in series. The inner diameters of the transmitting coil are $150 \mathrm{~mm}$ and $260 \mathrm{~mm}$ respectively, the outer diameters are $210 \mathrm{~mm}$ and $320 \mathrm{~mm}$ respectively, and the number of coil turns is 25 . The inner diameter of the receiving coil is 150 $\mathrm{mm}$, and the outer diameter is $210 \mathrm{~mm}$. The number of coil turns is 25 . The structure of asymmetrical coupling coil is shown in Fig. 8.

\subsection{Modeling of coupling coil}

In the improved asymmetric coupling coil model, the size of the transmitting coil is slightly larger than the size of the receiving coil, and the array form is used to improve the magnetic field uniformity and coil quality factor.

In order to analyze the performance of the proposed asymmetric coil structure, a three-dimensional simulation model of the asymmetric coil structure is established in ANSYS Maxwell, and the coil structure parameters are shown in Table 3.

Take the transmission distance of $40 \mathrm{~mm}$ as an example, the magnetic field distribution of asymmetric coupling coil structure is shown in Fig. 9.

From Fig. 9, it can be concluded that as the distance from the coil increases, the external magnetic field decays rapidly, and the magnetic field symmetrically distributes in the center of the coil. In the improved coil model, the magnetic field around the transmitting coil is stronger and more uniform. And the magnetic field distribution in the center area of the transmitting coil is more intensive. When the coupling coil axis has relative displacement, the coil is more conducive to power transmission.

The transient electric field solver in ANSYS Maxwell is used to solve the inductance value of two coil structures at a specific working frequency. When the resonance frequency is $162 \mathrm{KHz}$, this paper uses the transient solver to calculate the corresponding compensation capacitance. Then the ANSYS Simplorer software is used to simulate the circuit. The circuit simulation diagram is shown in Fig. 10.

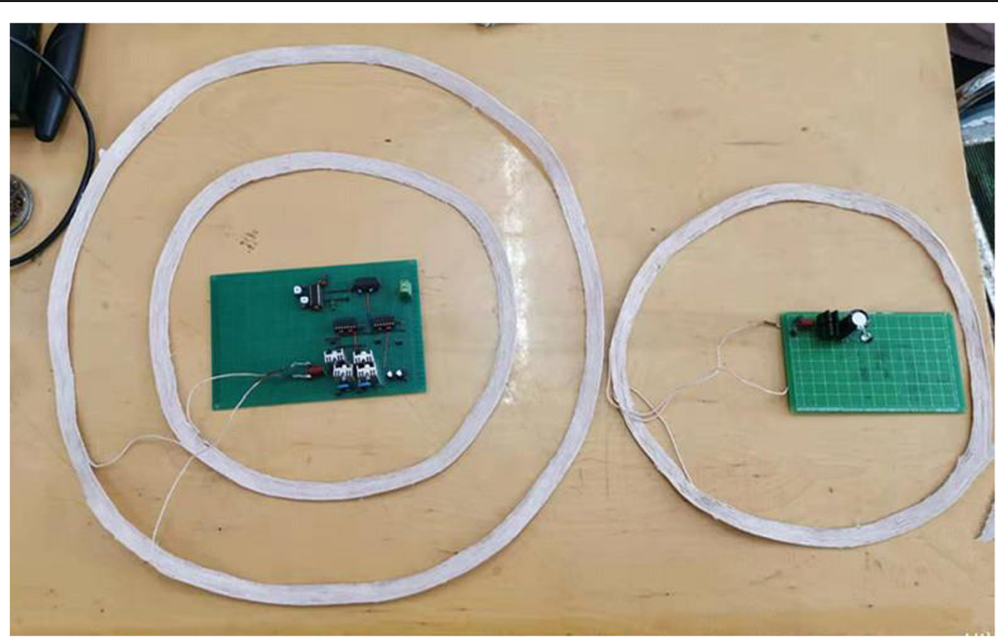

Fig. 11 Asymmetrical coupling coil 


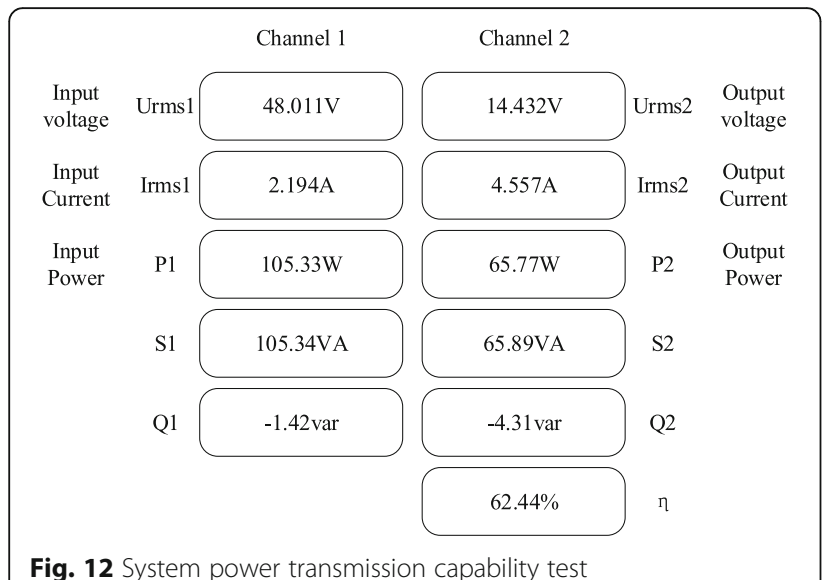

Fig. 12 System power transmission capability test

Compared with the traditional coil, the self-induction value of the improved transmitting coil is significantly increased, while the internal resistance is basically unchanged, so the quality factor of the coil is greatly improved. The receiving power varies with the coupling coefficient and driving frequency, but the transmission efficiency is always in a high transmission state. When the receiving power of the system reaches the maximum, the receiving power and transmission efficiency of the whole charging system reaches the maximum. Therefore, the WPT system transmits electric energy at the maximum receiving power point to improve the charging speed of UAV.

Combined with the solution results of system impedance parameters, this paper obtains the maximum receiving power, the maximum transmission efficiency, working frequency, coupling coil spacing, and quality factor through calculation, as shown in Table 4.

In conclusion, the improved asymmetrical coupling coil structure improves the quality factor of the coil, the strength, and uniformity of the magnetic field around the coil.

\section{Experimental results and analysis}

In order to test the working performance of the asymmetrical coupling coil, according to the above coil parameters, the coil is made of multi-strand Leeds wire winding, as shown in Fig. 11. In this paper, the WPT system is built with appropriate devices. Under the working conditions of $48 \mathrm{~V}$ input voltage, $2 \Omega$ load resistance, $162 \mathrm{kHz}$ working frequency, and basic alignment of coils, the system is tested. The output power of the system is $65.77 \mathrm{~W}$, and the efficiency from DC input side to load side is $62.44 \%$. The DC input side and the load side are detected by a power analyzer. In the meanwhile, the voltage and current values of the DC input side and the load side are obtained, as shown in Fig. 12. The experimental system can recognize wireless charging of UAV.

In order to verify that $162 \mathrm{kHz}$ is the optimal operating frequency of the coupling coil, we detect the power of the wireless charging system at frequencies of 130 $\mathrm{kHz}, 140 \mathrm{kHz}, 150 \mathrm{kHz}, 160 \mathrm{kHz}, 170 \mathrm{kHz}$, and 180 $\mathrm{kHz}$, and obtains the power of the wireless charging system at each frequency as shown in Fig. 13. From the figure, we can see that the power of the wireless charging system decreases with increasing frequency, proving that the higher the frequency, the smaller the power. Then, the efficiency of the wireless charging system with frequencies of $130 \mathrm{kHz}, 140 \mathrm{kHz}, 150 \mathrm{kHz}, 160 \mathrm{kHz}, 170$ $\mathrm{kHz}$, and $180 \mathrm{kHz}$ is tested, and the efficiency of the wireless charging system is obtained as shown in Fig. 14. It can be seen from the figure that the efficiency of the wireless charging system increases with increasing frequency, which proves that the higher the frequency, the greater the efficiency. We can see that with the increasing of working frequency, the maximum output power of the WPT system decreases and the efficiency of power transmission increases. When the working frequency of the system is $160 \mathrm{kHz}$, the maximum transmission power of the system is about $65.77 \mathrm{~W}$, and the power transmission efficiency can reach about $62.44 \%$. But in the experiment, when the frequency is $162 \mathrm{kHz}$,

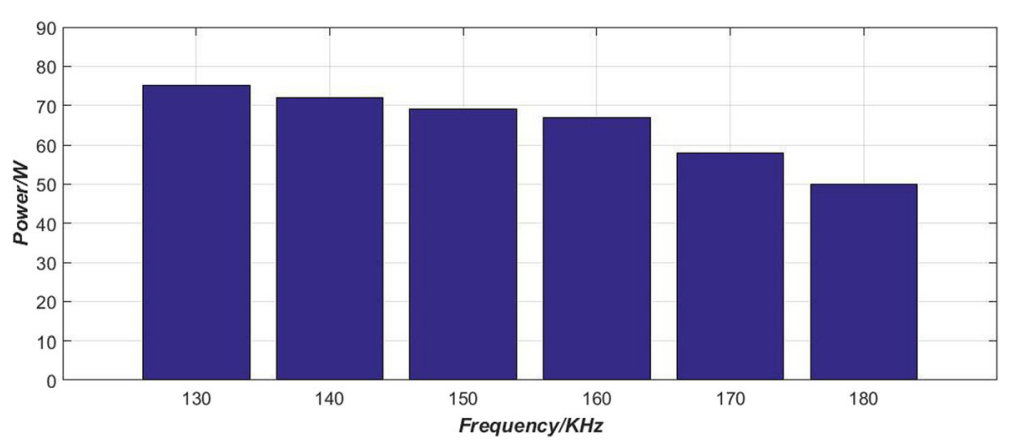

Fig. 13 The maximum output power of the wireless power transfer (WPT) system at different frequencies 


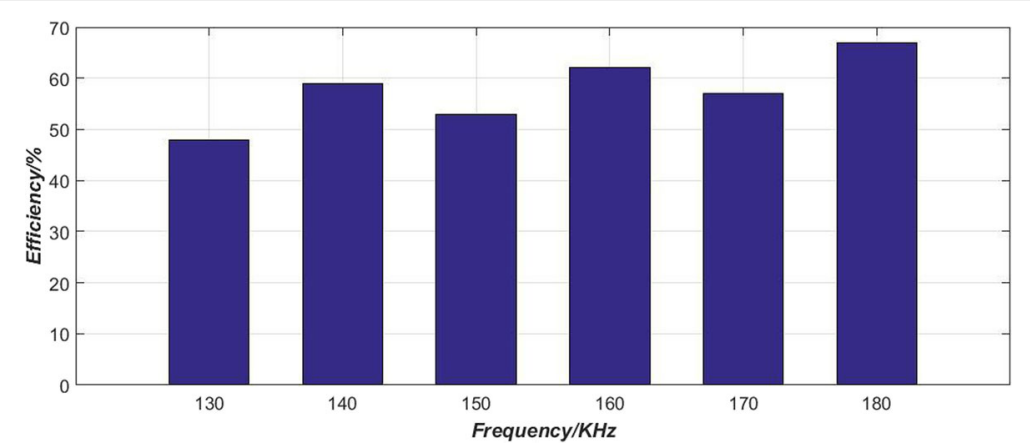

Fig. 14 The power transmission efficiency of the wireless power transfer (WPT) system at different frequencies

the charging performance of the system will be better than $160 \mathrm{kHz}$.

The driving waveform of the transmitter inverter circuit is shown in Fig. 15. When driving MOS transistor, the full bridge inverter must have a certain dead time; otherwise, it will cause a direct short circuit.

The load resistance will continuously change during the system charging process. Figure 16 is the test result of the system output voltage changes with the load. It is found that when the load resistance is changed, the output voltage of the system is still constant, which verifies the output characteristics of the constant voltage source of the system. The internal resistance of the coil is ignored during theoretical analysis which will lead to small load resistance and highly different measured value and theoretical value. In the early stage of charging, the equivalent load resistance value is small and the proportion of the internal resistance partial voltage of the coil is large, which results in a low output voltage. As the load resistance increases, the proportion of the internal resistance partial voltage of the system become smaller and smaller, and the measured values gradually approach to theoretical values.

When the coupling device is misaligned, the difference between the test and theoretical calculation results of the output voltage is very small, as shown in Fig. 17. In order to reduce the influence of the system internal resistance on the test results, the test is selected when the load is $20 \Omega$. The theoretical value in the figure is obtained by the simulation software. The results show that the test results are almost the same as the theoretical values, which proves that the established circuit model can be used to guide system parameter design. When the system is offset within the $[-40 \mathrm{~mm}, 40 \mathrm{~mm}]$ range of the $X$-axis and $Y$-axis, respectively, the system output voltage is above $10 \mathrm{~V}$. If the offset distance of the coupling device exceeds $40 \mathrm{~mm}$ and the output voltage is around $10 \mathrm{~V}$, a slight fluctuation in the system may cause the battery to fail to charge normally. The results prove that the wireless charging system can perform normal and stable charging when the offset between the $X$-axis and the $Y$-axis is $[-30 \mathrm{~mm}, 30 \mathrm{~mm}]$.

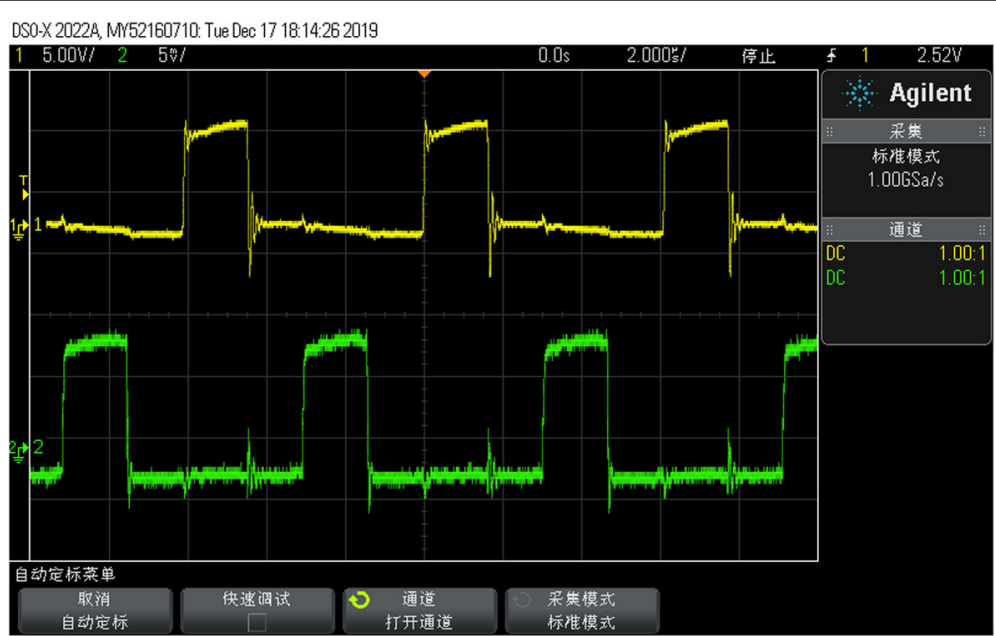

Fig. 15 Driving voltage waveform of MOS transistor 


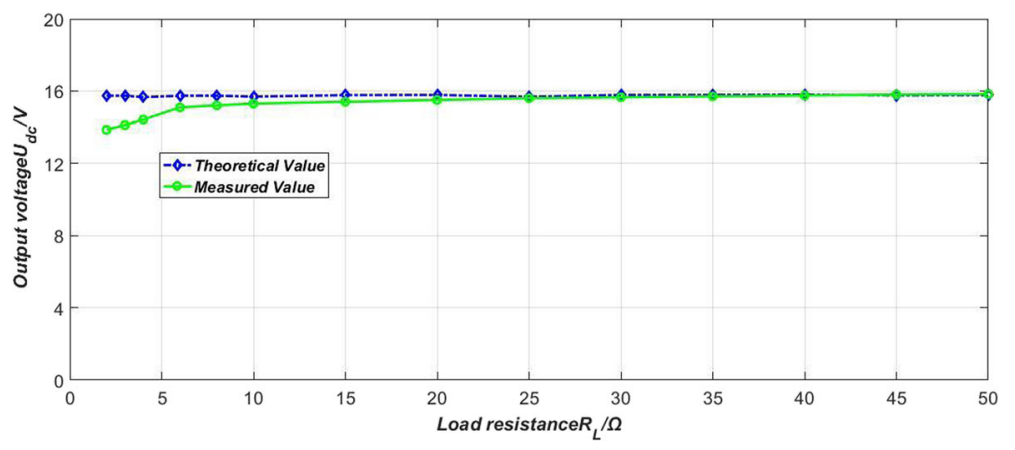

Fig. 16 Test results of output voltage with load resistance changes

The vertical distance between the primary coil and the secondary coil is also an important factor affecting the coupling coefficient in the WPT system. In other words, the distance between the installation positions of the coupling coils will directly affect the power transmission of the WPT system. Therefore, this experiment tests the transmission efficiency of the charging system at vertical distances of $10 \mathrm{~mm}, 20 \mathrm{~mm}, 30 \mathrm{~mm}, 40 \mathrm{~mm}$, and 50 $\mathrm{mm}$, as shown in Fig. 18.

From Fig. 18, the following conclusions can be drawn. In the offset range of $40 \mathrm{~mm}$, when the vertical distance exceeds $40 \mathrm{~mm}$, the transmission efficiency of the system is significantly lower than other charging efficiency, and the transmission efficiency decreases when the offset distance increases. When the vertical distance is less than $40 \mathrm{~mm}$, the transmission efficiency of the system will not be affected by the change of the offset distance, and will remain at a fixed value. If the vertical distance between the charging coils is $20 \mathrm{~mm}$, the error of vertical distance is allowed to be $20 \mathrm{~mm}$ in practice, which improves the stability of WPT system. Based on the above analysis, the vertical distance between the charging coils is finally determined to be $20 \mathrm{~mm}$.

In the WPT system, the maximum input power is about 105.33 W. The maximum output power can reach about $65.77 \mathrm{~W}$. The maximum charge current is about $4.557 \mathrm{~A}$. The power transmission efficiency of the system is about $62.44 \%$, which meets the charging demand of UAV.

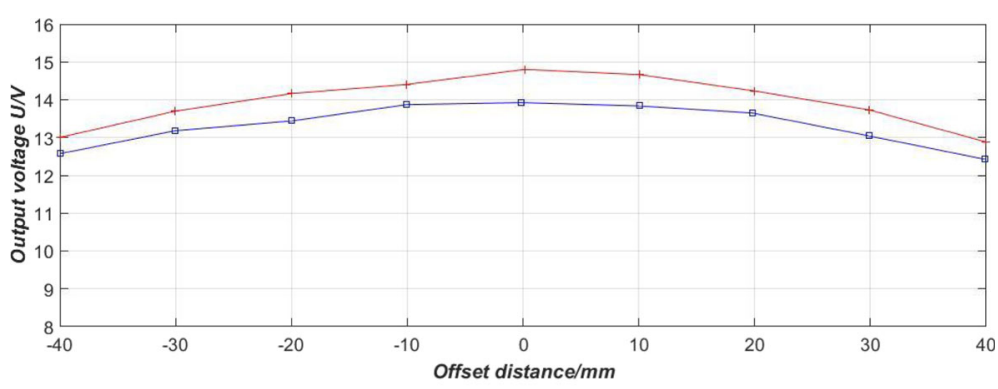

(a) $\mathrm{X}$-axis offset

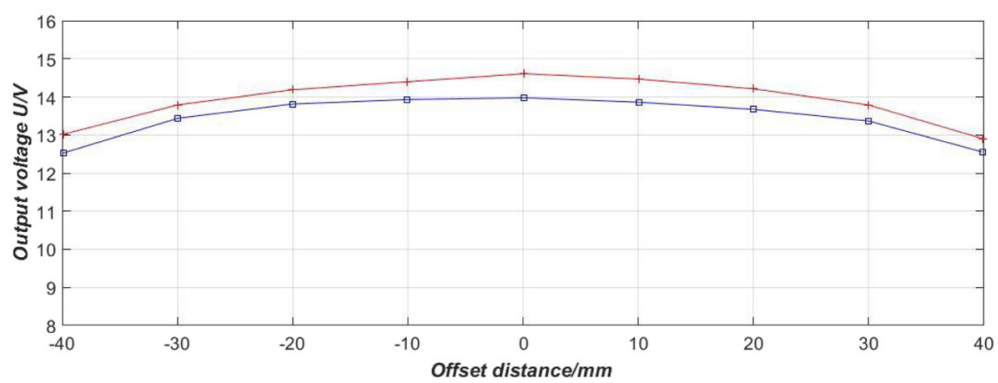

(b) Y-axis offset

Fig. 17 System output voltage variation diagram at offset. a $X$-axis offset. $\mathbf{b} Y$-axis offset 


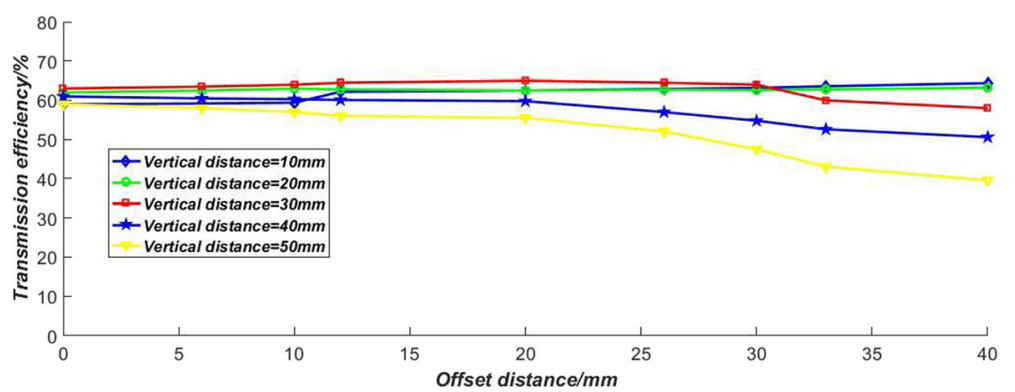

Fig. 18 The transmission efficiency of the WPT system at different vertical distances

\section{Conclusion}

In order to solve the problem of short endurance of the current lithium battery-powered UAV, a magnetic coupling resonance wireless charging system is designed in this paper. The wireless charging system mainly includes the transmitter inverter circuit, the coupling device, and the receiver rectifier circuit. According to the circuit model, the expressions of the receiving power and the transmission efficiency are derived to guide the parameter design of WPT system. Using ANSYS Maxwell to analyze the magnetic field of the coupling coil, we can know that the coupling coil can effectively restrain the magnetic field within $30 \mathrm{~mm}$ around the transmitting coil. The experimental results show that the selfinductance of the coupling coil changes little when it is offset, which proves that the system can solve the problem of low charging efficiency caused by the coil misalignment when the UAV lands. The system has the characteristics of constant voltage output, and the model established is correct.

\section{Abbreviations}

UAV: Unmanned aerial vehicle; WPT: Wireless power transmission; S-S: SeriesSeries; S-P: Series- Parallel; P-S: Parallel-Series; P-P: Parallel-Parallel; DC: Direct current; AC: Alternating current

\section{Acknowledgements}

The author wishes to thank all the colleagues for their efforts and efforts in this trial.

\section{Authors' contributions}

YY is the main author of the current paper. YY contributed to the development of the ideas, design of the study, theory, result analysis, and article writing. WS carried out the experimental work and the data collection and interpretation. XZ finished the analysis and interpretation of data and drafted the manuscript. WS and XZ conceived and designed the experiments, and undertook revision works of the paper. All authors read and approved the final manuscript.

\section{Funding}

This work is supported in part by the National Natural Science Foundation of China under Grants 51277043.

\section{Availability of data and materials}

Data sharing not applicable to this article as no datasets are generated or analyzed during the current study.

\section{Competing interests}

The authors declare that they have no competing interests.
Received: 27 December 2019 Accepted: 6 March 2020

Published online: 23 March 2020

\section{References}

1. Z. Huang, X. Xu, J. Ni, H. Zhu, W. Cheng, Multimodal representation learning for recommendation in Internet of Things. IEEE Internet Things J. 6-6, 10675-10685 (2019)

2. S. Pero, M. Sadko, V. Hrkvoje, The use of unmanned aerial vehicles for forest fire monitoring 2016, vol 93 (International Symposium Elmar, Zadar, 2016)

3. D. Lee, J. Zhou, W.T. Lin, Autonomous battery swapping system for quadcopter. In Proceedings of the 2015, vol 1-1 (International Conference on Unmanned Aircraft Systems (ICUAS), Denver, 2015), pp. 118-124

4. D. Ke, C. Liu, C. Jiang, F. Zhao, Design of an effective wireless air charging system for electric unmanned aerial vehicles, vol. 43 (2017)

5. T. Campi, S. Cruciani, F. Maradei, Wireless Power Transfer Technology Applied to an Autonomous Electric UAV with a Small Secondary Coil. Energies 11, 352 (2018)

6. G.A. Covic, J.T. Boys, Inductive power transfer. Proc. IEEE 101(6), 1276-1289 (2013)

7. T. Campi, S. Cruciani, F. Maradei, M. Feliziani, Near field reduction in a wireless power transfer system using LCC compensation. IEEE Trans. Electromag. Compat 59(2), 686-694 (2017)

8. T. Campi, F. Dionisi, S. Cruciani, V. de Santis, M. Feliziani, F. Maradei, Magnetic field levels in drones equipped with wireless power transfer technology, 2016. Asia-Pacific EMC Symp 17, 544-547 (2016)

9. L. Moraes, L.C. Carmo, R.F. Campos, M.A. Juca, L.S. Moreira, Autonomous Quadrotor for accurate positioning. IEEE Aerospace Elect. Syst. Mag. 32, 58$62(2017)$

10. H. Kim, C. Song, D.H. Kim, D.H. Jung, I.M. Kim, Coil design and measurements of automotive magnetic resonant wireless charging system for high-efficiency and low magnetic field leakage. IEEE Transac. Microwave Theory Techniques 64, 383-400 (2016)

11. S.H. Kang, J.H. Choi, F.J. Harackiewicz, C.W. Jung, Magnetic resonant threecoil WPT system between off/in-body for remote energy harvest. IEEE Microwave Wireless Comp. Lett. 26, 741-743 (2016)

12. T. Campi, S. Cruciani, G. Rodríguez, M. Feliziani, Coil design of a wireless power transfer charging system for a drone (2016 Conference on Electromagnetic Field computation (CEFC), 2016), pp. 1-1

13. H. Qiang, X.L. Huang, L.L. Tan, Maximum power transmission of inductively coupled radio power transmission system based on dynamic tuning. SCIENTIA SINICA Technologica 42(7), 830-837 (2012)

14. Z. Chen, H. Cai, Y. Zhang, C. Wu, M. Mu, Z. Li, M.A. Sotelo, A novel sparse representation model for pedestrian abnormal trajectory understanding. Expert Syst. Appl. 138, 1127 (2019)

15. S. Lee, S. Jeong, S. Hong, B. Sim, J. Kim, Design and analysis of EMI shielding method using intermediate coil for train WPT system (2018 IEEE Wireless Power Transfer Conference (WPTC), 2018), pp. 1-4

16. B. Wu, T.T. Cheng, T.L. Yip, Y. Wang, Fuzzy logic based dynamic decisionmaking system for intelligent navigation strategy within inland traffic separation schemes (Ocean Engineering, 2020), p. 197

17. Z. Huang, X. Xu, H. Zhu, M.C. Zhou, An efficient group recommendation model with multiattention-based neural networks. IEEE Transc. Neural Networks Learning Sys. (2020) 10, 1109 (2019)

18. T. Campi, S. Cruciani, M. Feliziani, Magnetic shielding of wireless power transfer systems in EMC14/Tokyo (2014) 
19. X. Liu, R. Zhu, B. Jalaian, Y. Sun, Dynamic spectrum access algorithm based on game theory in cognitive radio networks. Mobile Networks and Appl. 20(6), 817-827 (2015)

20. C. Song, D.H. Jung, E. Song, Y. Cho, S. Kim, Yuseong-gu, Electromagnetic interference reduction method from handheld resonant magnetic field charger (HH-RMFC) for electric vehicle (2014 IEEE Wireless Power Transfer Conference, 2014), pp. 5-8

21. J. Sallan, J.L. Villa, Optimal design of ICPT systems applied to electric vehicle battery charge. IEEE Trans. on Indust. Electronics 56(6), 2140-2149 (2009)

22. C. Song, Structure of handheld resonant magnetic coupling charger $(\mathrm{HH}-$ RMCC) for electric vehicle considering electromagnetic field. IEEE WPT 2013, 131-134 (2013)

23. T. Kan, R. Mai, P.P. Mercier, C.C. Mi, Design and analysis of a three-phase wireless charging system for lightweight autonomous underwater vehicles. IEEE Trans. Power Electron. 33(8), 6622-6632 (2018)

24. S. Kumar, Jayprakash, G.K. Mandavi, Wireless power transfer for unmanned aerial vehicle (UAV) charging. Int. Res. J. Engin. Technol. 4(8), 1939-1942 (2017)

25. T. Campi, S. Cruciani, M. Feliziani, F. Maradei, High efficiency and lightweight wireless charging system for drone batteries (2017 AEIT International Annual Conference, Cagliari, 2017), pp. 1-6

26. S.W. Kim, I.K. Cho, S.Y. Hong, Comparison of charging region differences according to receiver structure in drone wireless charging system (2017 International Conference on Information and Communication Technology Convergence, 2017), pp. 1058-1060

27. T. Campi, S. Cruciani, F. Maradei, M. Feliziani, Wireless charging system integrated in a small unmanned aerial vehicle (UAV) with high tolerance to planar coil misalignment (2019 EMC Sapporo/APEMC, Sapporo, 2019), pp. 601-604

28. F. Liu, Y. Yang, D. Jiang, X. Ruan, X. Chen, Modeling and optimization of magnetically coupled resonant wireless power transfer system with varying spatial scales. IEEE Trans. Power Electron. 32(4), 3240-3250 (2017)

29. P. Gao, Z. Tian, T. Pan, J. Wu, W. Cui, Transmission efficiency analysis and optimization of magnetically coupled resonant wireless power transfer system with misalignments. AlP Adv. 8(8) (2018)

30. D. Liu, H. Hu, S.V. Georgakopoulos, Misalignment sensitivity of strongly coupled wireless power transfer systems. IEEE Trans. Power Electron. 32(7), 5509-5519 (2017)

31. A. Barakat, K. Yoshitomi, R.K. Pokharel, Design approach for efficient wireless power transfer systems during lateral misalignment. IEEE Trans. Microw. Theory Techn 66(9), 4170-4177 (2018)

32. J.V.P.P. Dias, M. Miyatake, Increase in robustness against effects of coil misalignment on electrical parameters using magnetic material layer in planar coils of wireless power transfer transformer. Energies 11, 1970 (2018)

33. S.Y. Jeong, H.G. Kwak, G.C. Jang, S.Y. Choi, C.T. Rim, Dual-purpose nonoverlapping coil sets as metal object and vehicle position detections for wireless stationary EV chargers. IEEE Trans. Power Electron. 33(9), 7387-7397 (2018)

34. X. Liu, R. Zhu, A. Anjum, J. Wang, H. Zhang, M. Ma, Intelligent data fusion algorithm based on hybrid delay-aware adaptive clustering in wireless sensor networks. Futur. Gener. Comput. Syst. 104(1), 14 (2020)

\section{Publisher's Note}

Springer Nature remains neutral with regard to jurisdictional claims in published maps and institutional affiliations.

\section{Submit your manuscript to a SpringerOpen ${ }^{\circ}$ journal and benefit from:}

- Convenient online submission

- Rigorous peer review

- Open access: articles freely available online

- High visibility within the field

- Retaining the copyright to your article

Submit your next manuscript at $\boldsymbol{\nabla}$ springeropen.com 